Dictionaries define coercion as: 'the act of compelling by force of authority; compulsion'; 'the act, process, or power of coercing ... arm-twisting, force, compulsion, constraint, duress, pressure'; 'power based on the threat or use of force'; and so forth.

'If slavery is not wrong, nothing is wrong', declared Abraham Lincoln. Slavery is depriving a person of liberty because of who he is, not because of what he does or has done. If psychiatric slavery - involuntary mental hospitalisation - is not wrong, nothing is wrong. ${ }^{2}$

1 Newton-Howes G. Coercion in psychiatric care: where are we now, what do we know, where do we go? Psychiatrist 2010; 34: 217-20.

2 Szasz T. Coercion as Cure: A Critical History of Psychiatry. Transaction Publishers, 2007

Thomas Szasz is Professor of Psychiatry Emeritus, State University of New York Upstate Medical University, USA, email: tszasz@aol.com

doi: $10.1192 / p b .34 .12 .539 c$

\section{Why are psychosocial assessments following self-harm not completed?}

Mullins et al's study of accident and emergency (A\&E) presentations following self-harm added to the evidence for poor uptake of psychosocial assessments in the initial management of self-harm. ${ }^{1}$ Of particular concern was the finding that single men under 45 represented $39 \%$ of those not assessed. Although suicide rates among men in the UK fell between 1992 and 2007, the 2008 figures show a rise to 17.7 per 100000 , with highest rates seen in men aged $15-44 .^{2} \mathrm{~A}$ young man's presentation to A\&E following self-harm is a valuable opportunity to offer interventions which reduce his risk of repetition. The paradox is that with many of these opportunities being missed researchers cannot evaluate the effectiveness of interventions to reduce repetition in this group.

Those who discharge themselves from A\&E before completed assessment are 3 times more likely to repeat selfharm in the following year than those who are assessed. ${ }^{3}$ It is possible that impulsive personality traits are more heavily implicated than the lack of an assessment, but we need to know more about this group's behavioural characteristics so that we can learn how to engage them as soon as they present. From the Mullins et al study it is not clear whether patient factors or staff factors were more influential in determining completion of a psychosocial assessment. The National Institute for Health and Clinical Excellence (NICE) recommends that patients who self-harm are 'treated with the same care, respect and dignity as other patients', ${ }^{4}$ and reforms to medical and nursing training in some areas of the UK have managed to achieve cultural change. ${ }^{5}$ This is crucial because a humiliating or uncomfortable experience in $A \& E$ is likely to dissuade a patient from presenting should they self-harm again, and in cases of overdose this may increase mortality risk.

It is striking that of the 341 patients in Mullins et al's study who did not receive a psychosocial assessment, 141 (41\%) subsequently presented within the year of data collection having self-harmed, of whom 74 (52\%) slipped through the net a second time. We are unclear of the demographic characteristics of this subgroup, or whether there was a tendency for these individuals to leave $A \& E$ at the same stage in the referral process. However, if a study of this kind was repeated across a larger geographical area, it could be sufficiently powered to reveal valuable predictors which would help A\&E staff decide which patients to fast-track.

Finally, NICE recommendations on the communication of findings after self-harm assessments require auditing in future similar studies. A patient's general practitioner (GP) or community mental health team may remain completely unaware of their presentation to A\&E following self-harm unless a copy of the assessment is communicated to the relevant professionals. Even if the full psychosocial assessment was not performed, an outline of the presenting complaint would be of value. Armed with this information, a GP or key worker would be able to discern any patterns emerging in selfharm presentations, sometimes to many different hospitals, and would be in a unique position to manage apparent escalations in risk.

1 Mullins D, MacHale S, Cotter D. Compliance with NICE guidelines in the management of self-harm. Psychiatrist 2010; 34: 385-9.

2 Office for National Statistics. Suicides: UK Suicides Increase in 2008. ONS, 2010.

3 Crawford MJ, Wessely S. Does initial management affect the rate of repetition of deliberate self harm? Cohort study. BMJ 1998; 317: 985.

4 National Institute for Health and Clinical Excellence. Self-Harm: The Short Term Physical and Psychological Management and Secondary Prevention of Self Harm in Primary and Secondary Care. Clinical Guidelines 16. NICE, 2004

5 Pitman A, Tyrer P. Implementing clinical guidelines for self-harm highlighting key issues arising from the NICE guideline for self-harm. Psychol Psychother Theory Res Practice. 2008; 81: 377-97 (Special Issue: Implementing Clinical Guidelines in Everyday Practice).

Alexandra L. Pitman is MRC Research Fellow, Department of Mental Health Sciences, University College London, email: a.pitman@ medsch.ucl.ac.uk

doi: $10.1192 / \mathrm{pb} .34 .12 .540$

\section{Let's target screening more effectively}

I was very interested in the paper by Gumber et $a l_{,}^{1}$ which examined the monitoring of metabolic side-effects of antipsychotics in patients with schizophrenia. I commend them for their attempts to follow guidance for this monitoring and I agree that metabolic side-effects are important considerations for this group of patients. However, my critical review of the evidence of risk to patients with mental illness does not support the use of such widespread monitoring.

I will use the example of lipid monitoring to illustrate this. A large general practice study in the $\mathrm{UK}^{2}$ found that the relative risk of death from cardiovascular disease in people with mental illness when compared with controls was highest in younger people and reduced with age to a point that was not statistically significant in people over the age of 75 . The authors of that study claim that the three-fold increase in deaths for people under the age of 50 is the most worrying. This may be so, but the finding is worthy of closer scrutiny, especially when the implications for screening are being considered. In fact, the absolute risk of death from coronary heart disease in people with mental illness aged 18-49 was $0.1 \%$ over a median follow-up period of 4.7 years.

European guidelines for prevention of heart disease ${ }^{3}$ recommend monitoring of lipids only when the 10-year risk reaches $5 \%$ or more. It would seem difficult therefore to justify routine monitoring of mentally ill people aged 18-49. 
Also of concern is the lack of evaluation of harm to patients caused by what is essentially a screening programme of high-risk individuals. Such programmes are known to be associated with harm in a variety of forms. These include overdiagnosis, overtreatment and anxiety concerning the illness being investigated. ${ }^{4}$

Last, for a patient to give informed consent to participate in this kind of programme, they should be informed of the uncertainties inherent in it and the likelihood or otherwise of benefit to them of such a screening.

It is time to take stock and critically review which, if any, of these investigations are necessary for our patients.

1 Gumber R, Abbas M, Minajagi M. Monitoring the metabolic sideeffects of atypical antipsychotics. Psychiatrist 2010; 34: 390-5.

2 Osborn DP, Levy G, Nazareth I, Petersen I, Islam A, King MB. Relative risk of cardiovascular and cancer mortality in people with severe mental illness from the United Kingdom's General Practice Research Database. Arch Gen Psychiatry 2007; 64: 242-9.

3 Fourth Joint Task Force of the European Society of Cardiology and Other Societies on Cardiovascular Disease Prevention in Clinical Practice. European guidelines on cardiovascular disease prevention in clinical practice: executive summary. Eur J Cardiovasc Prevent Rehabil 2007; 14 (suppl 2): E1-40.

4 Jørgensen K, Gøtzsche P. Content of invitations for publicly funded screening: mammography. BMJ 2006; 332: 538-41.

Paul F. Reed is Consultant Psychiatrist with Lancashire Care NHS Foundation Trust, email: paul.reed@lancashirecare.nhs.uk

doi: 10.1192/pb.34.12.540a

\section{Scarcity of evidence base on management of acutely disturbed patients}

Brown et al give a useful insight into the practice at seven intensive care units all over the country. ${ }^{1}$

Their results show that $22 \%$ of patients were given rapid tranquillisation using the intramuscular route and $68 \%$ were not given any rapid tranquillisation medication at all.

The results table is confusing and the numbers do not add up; $3 \%$ appear not to have been given any medication at all, which causes concerns about the referral process to psychiatric intensive care units (PICUs) and whether patients were appropriately placed.

The study does not clarify the legal status of the patients and does not throw any light on the level of aggression of the patients in PICUs.

The most common diagnosis was schizophrenia/schizoaffective disorder (54\%), followed by mania (19\%) and substance misuse ( $8 \%$ ). The diagnosis for $19 \%$ of patients has not been provided in the study.

1 Brown S, Chhina N, Dye S. Use of psychotropic medication in seven English psychiatric intensive care units. Psychiatrist 2010; 34: 130-5.

Rupali Acharya CT2 Psychiatry, Trafford, Manchester, email: rupa_acharya@hotmail.com, Khurram Tanveer Sadiq Locum Consultant Psychiatrist, Trafford, Manchester

doi: $10.1192 / \mathrm{pb} .34 .12 .541$

\section{Authors' reply}

We are keen to encourage a wider discussion of the issues around the treatment of patients admitted to psychiatric intensive care units (PICUs) and welcome the opportunity to address points raised by Acharya \& Sadiq. In writing the paper ${ }^{1}$ we made a series of judgements about how best to present a large volume of data in an easily assimilated form and we are sorry if some of these decisions led to a lack of clarity.

One of the main findings of the study was that most PICU patients are safely managed without recourse to forced intramuscular (IM) medication, indeed that some patients are managed without any psychotropic medication at all. The study only collected data about treatment while the patients were in a PICU (this was a pragmatic decision as many patients came from and returned to distant units where data collection was not feasible). We suspect that some of the patients who did not receive any psychotropic medication in the PICU had received medication before transfer, possibly in the form of medium- or long-acting antipsychotic injection. Others will have received medication after transfer to the acute ward. The diagnoses of those patients who did not receive any medication were: schizophrenia (1), depression (2), druginduced psychosis (1), substance dependence (2), personality disorder (2), anxiety (1) and adjustment disorder (1).

The numbers in Table 1 do not always add up to $100 \%$ because some patients appear in several categories, for example: they were given IM rapid tranquillisation and IM zuclopenthixol acetate. All figures were rounded to the nearest $0.5 \%$; with this caveat we are confident that the appropriate figures (from text and table) do add up to $100 \%$.

The primary diagnoses of patients aggregated into the category 'other' were: learning (intellectual) disability, dementia, Asperger syndrome, obsessive-compulsive disorder, anxiety, adjustment disorder, and intoxication with drugs or alcohol.

We address the legal status of the patients and the level of behavioural disturbance more fully in a companion paper. ${ }^{2}$ With respect to the legal status of the patients, the findings were: 10 informal (3\%), 7 on Section 5(2) (2\%), 123 Section 2 (37\%), 158 Section 3 (48\%), 1 Section $4(<1 \%)$, 9 Section 37 (3\%), 19 a range of forensic sections covering different transfers from prison (6\%).

With respect to measurement of behavioural disturbance and mental state, we used the Brief Psychiatric Rating Scale (BPRS) and those subscales (hostility score, three-item Factor $\checkmark$ cluster and five-item hostility cluster) which focus on behavioural disturbance. The mean BPRS score fell from 58.2 on admission to 39.8 on transfer from PICU; the respective figures for the hostility score, Factor $V$ and hostility clusters were: 4.2 to $1.8,9.2$ to 5.5 and 17.3 to 11.1 .

We hope that these details clarify the points raised by Acharya \& Sadiq.

1 Brown S, Chhina N, Dye S. Use of psychotropic medication in seven English psychiatric intensive care units. Psychiatrist 2010; 34: 130-5.

2 Brown S, Chhina N, Dye S. The psychiatric intensive care unit: a prospective survey of patient demographics and outcomes at seven English PICUs. J Psychiatr Intensive Care 2008; 4: 17-27.

Steve Brown is Consultant Psychiatrist, Hampshire Partnership NHS Trust, email: Steve.Brown@hantspt-sw.nhs.uk

doi: $10.1192 / p b .34 .12 .541 a$

\section{Comment on the evaluation of the Time to Change anti-stigma campaign}

The study by Abraham et al suggests that a single exposure to selected Time to Change campaign material (those including the ' 1 in 4 ' message) delivered via post was not effective at improving attitudes towards people with mental illness. 